\title{
SODIUM ABSORPTION FROM THE EXOPLANETARY ATMOSPHERE OF HD 189733B DETECTED IN THE OPTICAL TRANSMISSION SPECTRUM ${ }^{1}$
}

\author{
Seth Redfield, ${ }^{2,3}$ Michael Endl, ${ }^{2}$ William D. Cochran, ${ }^{2}$ and Lars Koesterke ${ }^{2,4}$ \\ Received 2007 November 2; accepted 2007 December 5; published 2008 January 4
}

\begin{abstract}
We present the first ground-based detection of sodium absorption in the transmission spectrum of an extrasolar planet. Absorption due to the atmosphere of the extrasolar planet HD 189733b is detected in both lines of the Na I doublet. High spectral resolution observations were taken of 11 transits with the High Resolution Spectrograph (HRS) on the $9.2 \mathrm{~m}$ Hobby-Eberly Telescope (HET). The Na I absorption in the transmission spectrum due to HD $189733 \mathrm{~b}$ is $(-67.2 \pm 20.7) \times 10^{-5}$ deeper in the "narrow" spectral band that encompasses both lines relative to adjacent bands. The $1 \sigma$ error includes both random and systematic errors, and the detection is $>3 \sigma$. This amount of relative absorption in Na I for HD 189733b is 3 times larger than that detected for HD 209458b by Charbonneau et al. (2002) and indicates that these two hot Jupiters may have significantly different atmospheric properties.
\end{abstract}

Subject headings: atmospheric effects — line: profiles — planetary systems -

planets and satellites: individual (HD 189733b) — stars: individual (HD 189733) techniques: spectroscopic

\section{INTRODUCTION}

High signal-to-noise ratio $(\mathrm{S} / \mathrm{N})$ and high spectral resolution observations of transiting exoplanets provide an opportunity to measure the properties of exoplanet atmospheres and exospheres. Early theoretical models predicted that absorption, due to exoplanetary atmospheres, should be detectable in the strong resonance lines of $\mathrm{Na}$ I and other alkali metals in optical transmission spectra (Seager \& Sasselov 2000; Brown 2001). Charbonneau et al. (2002) used medium-resolution spectra $(R \equiv \lambda / \Delta \lambda \sim 5540)$ of Na I from the Space Telescope Imaging Spectrograph (STIS) on board the Hubble Space Telescope (HST) to make the first detection of absorption in the transmission spectrum caused by the exoplanetary atmosphere of HD $209458 \mathrm{~b}$ during transit. The measured Na I absorption, made in the "narrow" spectral window (5887-5899 $\AA$ ) since the doublet is not fully resolved, was $(-23.2 \pm 5.7) \times 10^{-5}$ deeper than in adjacent bands during transit and weaker than model predictions by a factor of $\sim 3$. Adjustments to cloud levels, metallicity, rainout of condensates, distribution of stellar flux, and photoionization of sodium could account for the discrepancy between the models and observations (Barman 2007; Fortney et al. 2003). Exospheric absorption was also detected for HD 209458b in several UV lines by Vidal-Madjar et al. (2003, 2004).

Previous ground-based attempts to detect absorption in the optical transmission spectra of HD 209458 and other bright transiting systems have measured only upper limits, likely due to limited observations of only a single effective transit (e.g., Bundy \& Marcy 2000; Moutou et al. 2001; Winn et al. 2004; Narita et al. 2005; Bozorgnia et al. 2006; Arribas et al. 2006). In addition, contaminants, such as telluric lines and, in some cases, the use of an iodine cell, hindered or prevented measurements of several spectral features, most notably the Na I lines.

Bouchy et al. (2005) announced the identification of the

\footnotetext{
${ }^{1}$ Based on observations obtained with the Hobby-Eberly Telescope, which is a joint project of the University of Texas at Austin, the Pennsylvania State University, Stanford University, Ludwig-Maximilians-Universität München, and Georg-August-Universität Göttingen.

${ }^{2}$ Department of Astronomy and McDonald Observatory, University of Texas, Austin, TX 78712; sredfield@ astro.as.utexas.edu.

${ }^{3}$ Hubble Fellow.

${ }^{4}$ Texas Advanced Computing Center, University of Texas, Austin, TX 78758 .
}

short-period $(P=2.2$ days $)$ transiting exoplanet around the bright $(V=7.7) \mathrm{K} 0 \mathrm{~V}$ star HD 189733. Subsequent work on this system tightly constrained the orbital parameters (Hébrard \& Lecavelier Des Etangs 2006; Bakos et al. 2006), determined that the axis of stellar rotation is aligned with the planetary orbital axis using the Rossiter-McLaughlin effect (Winn et al. 2006), found evidence for photometric stellar variability and a stellar rotational period of $\sim 12$ days (Henry \& Winn 2007; Winn et al. 2007), and identified transit signatures of starspots (Pont et al. 2007). Infrared studies of this system have been successful in characterizing the atmospheric temperature distribution (Deming et al. 2006; Knutson et al. 2007b).

\section{OBSERVATIONS AND DATA REDUCTION}

On the basis of the previous ground-based attempts to detect exoplanetary absorption in transmission spectra, our program strategy was designed to observe multiple transits and to specifically target the strong $\mathrm{Na}$ I lines. Observations of HD 189733 were taken over the course of a year (2006 August 112007 August 11) using the $9.2 \mathrm{~m}$ Hobby-Eberly Telescope (HET; Ramsey et al. 1998) High Resolution Spectrograph (HRS; Tull 1998). These observations cover the spectral range $5000-9000 \AA$, with a resolving power of $R \sim 60,000$. No iodine cell was used. A bright, rapidly rotating B star ( $\alpha$ Del) was observed immediately after HD 189733 in order to characterize the telluric absorption. We obtained spectra during 11 in-transit visits and 25 out-of-transit visits. Each visit is typically composed of several ( $\sim 3.2$ during transits and $\sim 5.1$ outside of transits) $600 \mathrm{~s}$ exposures, and the $\mathrm{S} / \mathrm{N}$ at $\mathrm{Na}$ I of each exposure is $\sim 320$ per resolution element. Observations are considered intransit if the entire exposure is obtained within first and fourth contacts.

The data were reduced using Image Reduction and Analysis Facility (IRAF; Tody 1993) and Interactive Data Language (IDL) routines to subtract the bias, flat-field the images, remove scattered light and cosmic-ray contamination, extract the echelle orders, calibrate the wavelength solution, and convert to heliocentric velocities. Local interstellar medium (LISM) and stellar lines were removed from the telluric standard before being applied to the target spectra. LISM Na I absorption is detected in the spectra of $\alpha \operatorname{Del}(\sim 74 \mathrm{pc})$, our telluric standard, 

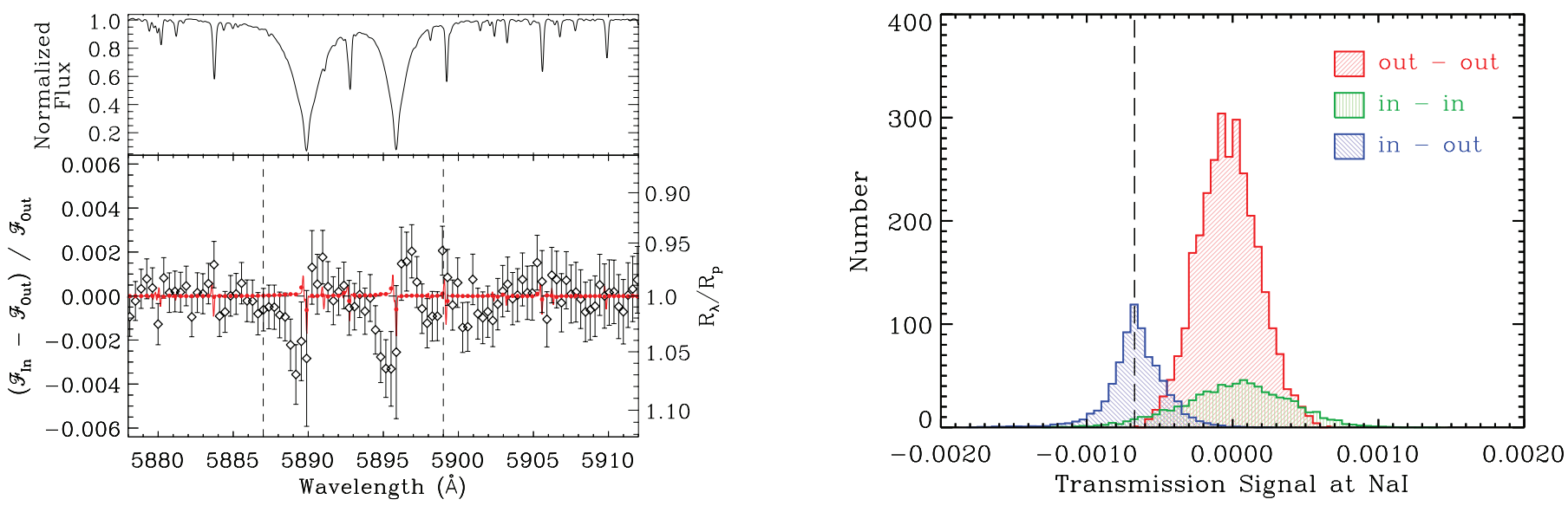

FIG. 1.-Top left: Spectrum of HD 189733 near the Na I doublet. Bottom left: The difference of the relative flux of the in-transit template $\left(\mathcal{F}_{\text {in }}\right)$ and the outof-transit template $\left(\mathcal{F}_{\text {out }}\right)$. Excess absorption in the in-transit template is clearly detected in both $\mathrm{Na}$ I lines. Also shown is the contribution of differential limb darkening (red line and filled circles), which has been removed from the data, but the effect is minimal. The vertical dashed lines indicate the narrow bandpass used to calculate the relative transmission spectrum absorption and is identical to that used by Charbonneau et al. (2002). The right-hand axis gives the effective radius of the planet as a function of wavelength, assuming stellar and planetary properties from Pont et al. (2007). Right: Distributions of the empirical Monte Carlo analysis, which demonstrate the stability of the observations and the contribution of systematic errors. The full data set transmission spectrum absorption measurement is indicated by the vertical dashed line. Given the random and systematic errors measured by the standard deviation of the "out-out" distribution, this exoplanetary absorption detection is $>3 \sigma$.

consistent with LISM detections toward other nearby stars (Redfield \& Linsky 2002) and previous Ca II and Na I observations of $\alpha$ Del by Vallerga et al. (1993) and Welsh et al. (1991). Stellar lines in our telluric standard, a rapidly rotating hot B star, are very weak and were removed using a stellar model (Koesterke et al. 2007; Fitzpatrick \& Massa 2005; Castelli \& Kurucz 2006). Observations of HD 189733 were divided by the cleaned telluric standard spectra to remove the contaminating telluric features.

To create the in-transit and out-of-transit templates, additional processing was required to match the blaze response and wavelength calibration of all observations. Over the short spectral windows $(\sim 30 \AA)$ of interest, fluctuations in the blaze response are smooth and slowly varying and were easily characterized by a low-order polynomial. Likewise, precise spectral alignment required small adjustments ( $\sim 0.1-0.2$ pixels) to the wavelength solution based on the cross correlation of nearby stellar lines.

\section{Na I TRANSMISSION ABSORPTION}

Figure 1 shows the observed Na I region of the stellar spectrum. The doublet is fully resolved and accompanied by many weak stellar lines. The bottom left plot shows the transmission spectrum, or the difference of the relative fluxes, $\left(\mathcal{F}_{\text {in }}-\right.$ $\left.\mathcal{F}_{\text {out }}\right) / \mathcal{F}_{\text {out }}$, of the ultrahigh $\mathrm{S} / \mathrm{N}$ in-transit template $\left(\mathcal{F}_{\text {in }}\right.$; $\mathrm{S} / \mathrm{N} \sim 1600)$ and the even higher $\mathrm{S} / \mathrm{N}$ out-of-transit template $\left(\mathcal{F}_{\text {out }} ; \mathrm{S} / \mathrm{N} \sim 3400\right)$. The signal predicted due to differential stellar limb darkening is shown and has been removed from the observations, although the effect is minimal.

Excess absorption is clearly detected in the in-transit spectrum $\left(\mathcal{F}_{\text {in }}\right)$ compared with the out-of-transit spectrum $\left(\mathcal{F}_{\text {out }}\right)$ for both $\mathrm{Na}$ I lines. Using the same "narrow" spectral region defined by Charbonneau et al. (2002), 5887-5899 A, we measure an excess of absorption $(-67.2 \pm 7.2) \times 10^{-5}$, when compared with the adjacent spectrum. This is $\sim 3$ times larger than that measured for HD 209458b $\left([-23.2 \pm 5.7] \times 10^{-5}\right.$; Charbonneau et al. 2002). We measure a random error of $7.2 \times$ $10^{-5}$, comparable to that achieved by the HST/STIS observations.
We also analyze a neighboring strong stellar line that is predicted to show no significant exoplanetary atmospheric absorption. In Figure 2, we present the same analysis near the $\mathrm{Ca}$ I line at $6122 \AA$. No absorption is detected in this region, and over a narrow spectral range of 6119-6125 $\AA$ we measure an insignificant amount of emission $(+15.4 \pm 6.8) \times 10^{-5}$, consistent with there being no difference between the in-transit and out-of-transit templates.

\subsection{Stellar Limb Darkening}

Differential limb darkening, the relative difference in the limb-darkening response of the cores of spectral lines and the continuum, contributes to the transmission spectrum. Since we are dealing with a narrow spectral window and the spectra are normalized by the adjacent continuum, these observations are not sensitive to the broadband color dependency expected due to limb darkening (Brown et al. 2001; Ballester et al. 2007).

Essentially, the in-transit template is a collection of spectra where a small fraction ( 2.5\%; Pont et al. 2007) of the stellar disk is blocked. This blocked component causes a loss of flux at a particular radial velocity and leads to the Rossiter-McLaughlin effect (Winn et al. 2006). In addition, the blocked component is associated with a specific $\mu$-angle and therefore causes the loss of a specifically limb-darkened fraction of the stellar disk. The observational distribution of $\mu$-angles, all necessarily $<0.73$ since the HD $189733 \mathrm{~b}$ transits at an angle slightly inclined from precisely edge-on $\left(i=85.7^{\circ}\right.$; Pont et al. 2007), will produce a unique differential limb-darkening signature. Indeed, with sufficient $\mathrm{S} / \mathrm{N}$, a transiting exoplanet could be used as a blocking obstruction, where the resulting differential limb-darkening spectrum would constrain, among other things, the temperature profile of the stellar atmosphere. In our case, we are concerned about the contribution this signal may have on the absorption feature in the transmission spectrum.

We use a stellar model of HD 189733, based on literature values, $T_{\text {eff }}=5050 \mathrm{~K}, \log g=4.5$, and $\mathrm{Fe} / \mathrm{H}=-0.03$ (Bouchy et al. 2005), to calculate the stellar flux blocked by the exoplanet for each observation (Koesterke et al. 2007). We assume a $0.755 R_{\odot}$ star and a $1.154 R_{\text {Jup }}$ planet (Pont et al. 

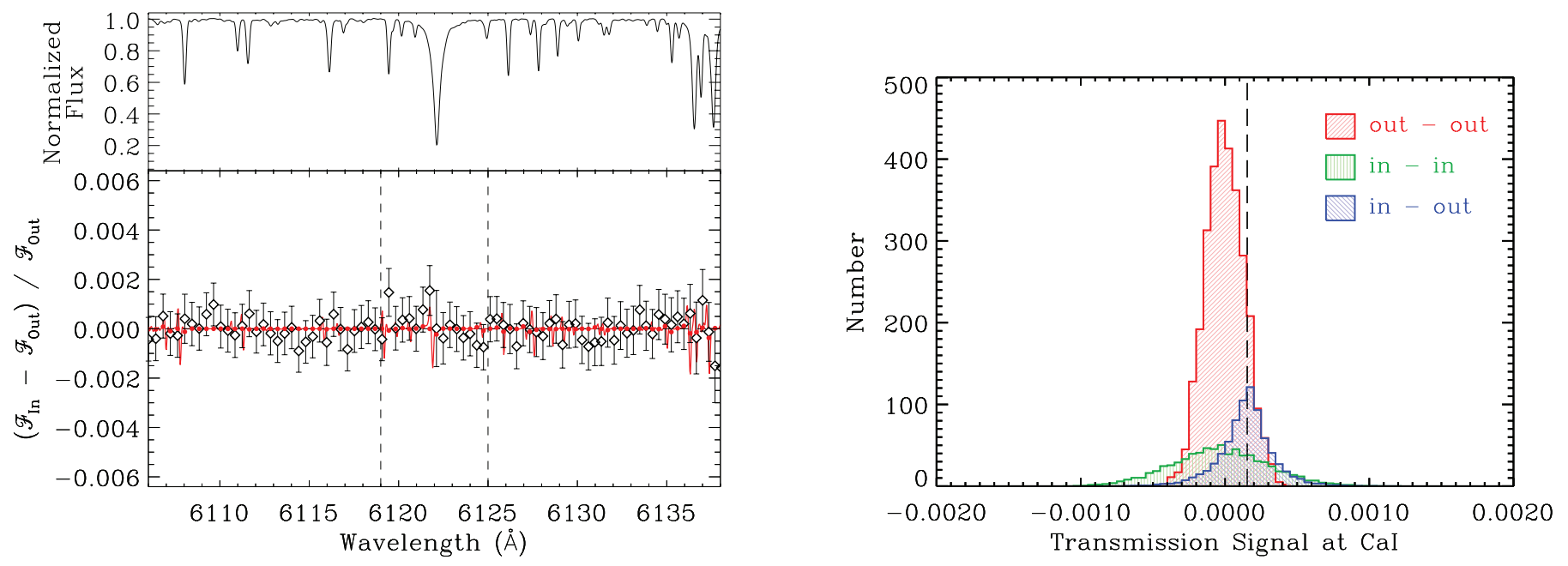

FIG. 2.-Same as Fig. 1, but for the $\mathrm{Ca}$ I line, which like Na I is a strong line but predicted not to show absorption in the transmission spectrum.

2007). The blocked flux is subtracted from the integrated disk flux and the continuum is normalized. The colored spectrum in the bottom panel of the left plot in Figures 1 and 2 shows the relative difference spectrum due to differential limb darkening for our distribution of in-transit observations. The contribution of differential stellar limb darkening is much smaller than the observed absorption, and as for HD 209458 (Charbonneau et al. 2002), at $\mathrm{Na}$, it is actually a net emission, where it contributes $+1.46 \times 10^{-5}$ in the "narrow" spectral window. At $\mathrm{Ca}$ I the contribution is $-0.10 \times 10^{-5}$. In both cases, the differential limb darkening contribution is well below the $1 \sigma$ random error.

\subsection{Empirical Monte Carlo}

Given the weakness of any atmospheric absorption in a transmission spectrum, it is critical to assess the contribution of systematic errors. Systematic errors associated with data reduction may arise from the continuum normalization, wavelength calibration, removal of the stellar and interstellar lines from the telluric standard, and the differential limb-darkening calculation. In addition, there are systematic contributions that are unrelated to data reduction procedures, including stellar variability, the changing distribution of starspots, and transits over active regions. These issues are particularly important since HD 189733 is a moderately active star that is known to exhibit photometric variability due to starspots (Henry \& Winn 2007; Pont et al. 2007). Furthermore, because our data set is composed of many observations, spread over a year, it is important to quantify the stability of the individual observations that contribute to our in-transit and out-of-transit templates.

We have constructed a diagnostic that we call an empirical Monte Carlo, or bootstrapping, analysis. It involves randomly selecting from the $\sim 200$ individual exposures a test sample of "in-transit" exposures, running through our entire data analysis algorithm, and measuring any signal in the transmission spectrum. We repeat this exercise thousands of times in order to establish statistical significance. Three different scenarios are explored: (1) an "out-out" comparison, where a subset of outof-transit exposures, equal to the number of our in-transit exposures $(\sim 36)$, is selected and compared with the rest of the out-of-transit exposures; (2) an "in-in" comparison, where a subset of in-transit exposures $(\sim 8)$, set at the same ratio as the number of in-transit exposures to the number of out-of-transit exposures (36/165), is compared with the rest of the in-transit exposures; and (3) an "in-out" comparison, where an increasing number of in-transit exposures (up to half of the sample) is removed from the in-transit template and compared with the out-of-transit template. The distribution of the "narrow" band difference of the relative flux measurements for all realizations of the three scenarios are shown for $\mathrm{Na} \mathrm{I}$ in the right panel of Figure 1 and for $\mathrm{Ca} \mathrm{I}$ in Figure 2.

Both the "in-in" and "out-out" distributions are centered on zero net signal, indicating that there is no significant difference between the different out-of-transit exposures ("out-out" scenario) and no significant difference between the various in-transit exposures ("inin" scenario). Since we have fewer in-transit exposures in order to do an "in-in" comparison, the $\mathrm{S} / \mathrm{N}$ is greatly reduced, and the distribution is much wider than the "out-out" distribution. For $\mathrm{Na}$ I, the mean value $(\mu)$ of the "out-out" distribution is $-3.5 \times$ $10^{-5}$ with a standard deviation $(\sigma)$ of $20.7 \times 10^{-5}$, whereas for the "in-in" distribution, $\mu=3.9 \times 10^{-5}$ and $\sigma=35.5 \times 10^{-5}$. The results are similar for the $\mathrm{Ca}$ I line, where for the "out-out" distribution $\mu=-0.5 \times 10^{-5}$ and $\sigma=13.7 \times 10^{-5}$, and for the "in-in" distribution $\mu=-4.0 \times 10^{-5}$ and $\sigma=32.8 \times 10^{-5}$. The $\mathrm{Ca}$ I line is located more centrally on the blaze and therefore is acquired with slightly higher $\mathrm{S} / \mathrm{N}$, which translates into a narrower distribution of measurements.

As expected, the "in-out" distributions are centered on the value we obtained from the full data set. The width of this distribution provides a consistency check that a handful of intransit exposures are not dominating the measurement. This demonstrates, along with the "in-in" distribution being centered at zero, that all the in-transit exposures, over 11 transits, consistently show excess absorption from the transiting exoplanetary atmosphere.

The width of the "out-out" distribution measures directly the propagation of systematic errors through our analysis. These realizations include the contribution of both reduction systematics, as well as any astrophysical systematics that are not correlated with the exoplanet orbital period. For example, if changing distributions of starspots (i.e., different phases of the stellar variability cycle) could mimic an absorption feature in the transmission spectrum, we would expect to see a significant fraction of "out-out" realizations result in such measurements. 
Because no such realizations are able to replicate the level of the detection, and because the orbital period is completely decoupled from the stellar variability period (Henry \& Winn 2007) such that our 11 in-transit visits are randomly distributed in stellar rotational phase, stellar variability cannot be responsible for the observed absorption in the transmission spectrum. The standard deviation of our "out-out" distribution is adopted as the appropriate $1 \sigma$ error for our transmission spectrum measurements since it encompasses both random and systematic errors. Our $\mathrm{Na}$ I absorption detection is $(-67.2 \pm 20.7) \times$ $10^{-5}$, while the signal at $\mathrm{Ca} \mathrm{I}$ is $(+15.4 \pm 13.7) \times 10^{-5}$.

\section{PROPERTIES OF THE HD 189733B ATMOSPHERE}

The absorption feature in the transmission spectrum in Figure 1 is a direct measurement of the effective radius of the exoplanetary atmosphere due to the high opacity at the center of the strong Na I lines. The right axis of Figure 1 shows the magnitude of the absorption in units of exoplanetary radius. The cores of the $\mathrm{Na}$ I lines extend to effective radii of $\sim 1.06 R_{\mathrm{pl}}$.

The spectra have not been corrected for the Doppler shift of the exoplanetary absorption. During conjunction (e.g., midtransit), the radial velocity of the planet is $0 \mathrm{~km} \mathrm{~s}^{-1}$, although the rate of change in radial velocity is at its maximum. Over the duration of the transit, the radial velocity of HD $189733 \mathrm{~b}$ will change by $\pm 19.2 \mathrm{~km} \mathrm{~s}^{-1}$. This will cause a smearing of the absorption profile. The observed feature appears to be significantly blueshifted from the stellar line center, by $\sim 38 \mathrm{~km}$ $\mathrm{s}^{-1}$, and, if astrophysical in origin, may be a combination of the planetary orbital motion and high-speed winds flowing from the hot dayside, predicted by several dynamical atmospheric models (e.g., Cho et al. 2003; Cooper \& Showman 2005).

Recent infrared observations of HD 209458b by Knutson et al. (2007a) show evidence for a temperature inversion in the upper atmosphere of that exoplanet. Burrows et al. (2007) presented accompanying models that require an additional absorber in the upper atmosphere of HD 209458b, which could be high-altitude clouds and explain the weaker than expected $\mathrm{Na}$ I absorption signal in the transmission spectrum (Charbonneau et al. 2002; Fortney et al. 2003). Our measurement of strong $\mathrm{Na}$ I absorption argues against the presence of such clouds high in the atmosphere of HD $189733 \mathrm{~b}$, consistent with the isothermal temperature profile presented for HD $189733 \mathrm{~b}$ (Tinetti et al. 2007).

\section{CONCLUSIONS}

We present the first ground-based detection of absorption in the transmission spectrum of a transiting extrasolar planet. The detection is based on the first high-resolution $(R \sim 60,000)$ transmission spectrum, with the $\mathrm{Na}$ I doublet fully resolved. The Na I transmission spectrum absorption for HD 189733b, measured in a "narrow" spectral band that encompasses both lines, is $(-67.2 \pm 20.7) \times 10^{-5}$. The estimated error includes both random and systematic contributions. The amount of absorption in $\mathrm{Na}$ I due to HD $189733 \mathrm{~b}$ is $\sim 3$ times larger than that detected for HD 209458b by Charbonneau et al. (2002) and indicates that the two exoplanets may have significantly different atmospheric properties. The excess absorption appears in a blueshifted component, while the red half of the $\mathrm{Na}$ I doublet lines has a net deficit of absorption during transit. $\mathrm{Fu}-$ ture work will include the analysis of other important optical lines (e.g., $\mathrm{K} \mathrm{I}, \mathrm{H} \alpha$ ), detailed analysis of the absorption profile and comparison with atmospheric models, measurements of spectral indicators of stellar variability, and observations of other transiting systems. High-resolution space-based spectroscopic observations will provide a reliable confirmation of this detection and access to important lines in the ultraviolet. Transmission spectra not only have the potential to probe the physical properties of hot-Jupiter and hot-Neptune exoplanets but lay the foundation toward detailed physical diagnostics of Earthsized extrasolar planetary atmospheres (Ehrenreich et al. 2006).

The authors would like to thank the referee, Ronald Gilliland, for his insightful comments. S. R. would like to acknowledge support provided by the Hubble Fellowship grant HST-HF01190.01 awarded by the STScI, which is operated by the AURA, Inc., for NASA, under contract NAS 5-26555. M. E. and W. D. C. acknowledge support provided by NASA under grants NN05G107G and NNX07AL70G issued through the TPF Foundation Science and the Origins of Solar Systems programs. The Hobby-Eberly Telescope (HET) is a joint project of the University of Texas at Austin, the Pennsylvania State University, Stanford University, Ludwig-Maximilians-Universität München, and Georg-August-Universität Göttingen. The HET is named in honor of its principal benefactors, William P. Hobby and Robert E. Eberly.

Facilities: HET(HRS)

\section{REFERENCES}

Arribas, S., et al. 2006, PASP, 118, 21

Bakos, G. Á., et al. 2006, ApJ, 650, 1160

Ballester, G. E., Sing, D. K., \& Herbert, F. 2007, Nature, 445, 511

Barman, T. 2007, ApJ, 661, L191

Bouchy, F., et al. 2005, A\&A, 444, L15

Bozorgnia, N., et al. 2006, PASP, 118, 1252

Brown, T. M. 2001, ApJ, 553, 1006

Brown, T. M., et al. 2001, ApJ, 552, 699

Bundy, K. A., \& Marcy, G. W. 2000, PASP, 112, 1421

Burrows, A., et al. 2007, ApJ, in press (arXiv: 0709.3980)

Castelli, F., \& Kurucz, R. L. 2006, A\&A, 454, 333

Charbonneau, D., et al. 2002, ApJ, 568, 377

Cho, J. Y.-K., et al. 2003, ApJ, 587, L117

Cooper, C. S., \& Showman, A. P. 2005, ApJ, 629, L45

Deming, D., et al. 2006, ApJ, 644, 560

Ehrenreich, D., et al. 2006, A\&A, 448, 379

Fitzpatrick, E. L., \& Massa, D. 2005, AJ, 129, 1642

Fortney, J. J., et al. 2003, ApJ, 589, 615

Hébrard, G., \& Lecavelier Des Etangs, A. 2006, A\&A, 445, 341

Henry, G. W., \& Winn, J. N. 2007, ApJ, submitted (arXiv: 0709.2142)

Knutson, H. A., et al. 2007a, ApJ, in press (arXiv:0709.3984)
Knutson, H. A., et al. 2007b, Nature, 447, 183

Koesterke, L., Allende Prieto, C., \& Lambert, D. L. 2007, ApJ, submitted

Moutou, C., et al. 2001, A\&A, 371, 260

Narita, N., et al. 2005, PASJ, 57, 471

Pont, F., et al. 2007, A\&A, submitted (arXiv: 0707.1940)

Ramsey, L. W., et al. 1998, Proc. SPIE 3352, 34

Redfield, S., \& Linsky, J. L. 2002, ApJS, 139, 439

Seager, S., \& Sasselov, D. D. 2000, ApJ, 537, 916

Tinetti, G., et al. 2007, Nature, 448, 169

Tody, D. 1993, in ASP Conf. Ser. 52, Astronomical Data Analysis Software and Systems II, ed. R. J. Hanisch, R. J. V. Brissenden, \& J. Barnes (San Francisco: ASP), 173

Tull, R. G. 1998, Proc. SPIE 3355, 387

Vallerga, J. V., et al. 1993, ApJ, 411, 729

Vidal-Madjar, A., et al. 2003, Nature, 422, 143 . 2004, ApJ, 604, L69

Welsh, B. Y., et al. 1991, ApJ, 381, 462

Winn, J. N., et al. 2004, PASJ, 56, 655

2006, ApJ, 653, L69

2007, AJ, 133, 1828 